\title{
Using On-line Guided-Self Determination to Provide High Quality Diabetes Self-Management: An Analysis with Activity Theory
}

\author{
Nilmini Wickramasinghe \\ Epworth HealthCare \& Deakin University, Australia \\ nilmini.work@gmail.com \\ Freimut Bodendorf \\ Friedrich Alexander University,Germany \\ freimut.bodendorf@fau.de
}

\author{
Bodil Rasmussen \\ Deakin University, Australia \\ bodil.rasmussen@deakin.edu.au
}

\author{
Judy Currey \\ Deakin University, Australia \\ judy.currey@deakin.edu.au
}

\begin{abstract}
This paper presents and evaluation of the use of a developed on line guided Self-Determination (GSD) solution for young adults with Type 1 diabetes. Activity theory is proffered as a suitable analysis lens to highlight and unpack key social interactions. An exploratory descriptive design with four stages that involved: (1) developing the GSD program online; (2) training diabetes educators to use the GSD program in an online format; (3) implementing and pilot testing the GSD program; and $(d)$ evaluating the online version formed the adopted methodology.
\end{abstract}

Key words: Activity theory, Self-Determination; Diabetes, self-management, online education, empowerment, Life-skills

\section{Introduction}

Living with Type 1 diabetes requires lifelong selfcare, achieving tight blood glucose management, to optimise well-being and prevent complications. Psychosocial stress, the ability to adjust to change and coping ability influence a person's selfmanagement potential and consequently metabolic control and impact on clinical outcomes and mental health [1-3]. Further, living with diabetes requires constant discipline. People with diabetes benefit significantly from access to timely, targeted and personalised information [4]. On average, a person with diabetes will be in health professionals' care for ten hours in a year. For the rest of the year, people with diabetes are on their own [4]; hence learning to self-manage diabetes is vital for good health outcomes. Current education interventions to achieve glycaemic control are not successful if people lose motivation to self-manage their diabetes [5]. Inadequate glycaemic control is frequently associated with lack of motivation [6] when psychological and social factors impact on people's lives [7, 8].
Social interactions and communication with health professionals play an integral role in empowering people with diabetes to self-management outcomes $[9,10]$ hence awareness of social interactions is pertinent. Activity theory, a socio-technical theory is a helpful framework to assist in identifying shortcomings or contradiction in social interactions coupled with technology use.

Motivation to manage diabetes is particularly affected when people are experiencing life changes, such as moving out of home, starting university and entering the workforce. These changes can adversely affect decision making when managing diabetes $[11,12]$. Younger adults, aged 18-40 years have numerous life changes, often simultaneously. Changes in life patterns affect diabetes management, hence people need to make complex decisions during transitional periods.

\section{Background}

The Guided Self Determination (GSD) method, developed by Zoffmann [5] improves the life skills of young adults with diabetes [2,6]. Life skills are "those personal, social, cognitive and physical skills that enable people to control and direct their lives, and to develop the capacity to live with and produce change in their environment" [5].

The GSD method was designed to guide both participants with persistent inadequate glycaemic control and professionals managing participants with diabetes using mutual reflection. Participants are prompted to systematically explore and express their personal difficulties and experiences with diabetes through words and drawings on shared worksheets, which in turn enable people to discover their potential for change [13]. Reflections are recorded on these worksheets designed to assist young adults to express their views and prepare them to actively participate in the care process [6].

Suboptimal diabetes self-management can result in serious complications in people with type 1 diabetes (T1DM). Young adults with diabetes report current health services in rural and regional 
area do not meet their information and support needs related to access issues, travel/time constraints and limited choice of health professionals [14].

Novel strategies, for example, online health services are required to assist those with limited access to health care. Technology can supplement current care by providing educational and motivational support [15]. A national survey of Australians aged 15 years and older (including 96\% of 18-24 year olds) showed $98 \%$ of respondents had internet access and $52 \%$ of those with internet access used the internet daily [16]. Increased internet access via mobile devices has also increased user access to internet services and creates opportunities for health professionals to leverage this mode of communication.

The presented pilot study describes the development of an online interactive version of GSD for young people with diabetes. The specific study aims were to engage young people with diabetes who have low motivation and inadequate blood glucose control in a specifically tailored GSD program online, and to explore the perspectives of the young people and the diabetes educator participants regarding the feasibility and utility of the online GSD program.

Finally, we use activity theory as a lens to assist the assessment of the program and the online solution .

\section{Principles of Activity Theory}

Activity theory, first developed by Leontiev, is a rich theory to assist with understanding social dynamics [17-22]. The concept of activity reflects a special type of relationship between the subject and the object [17]. This relationship is defined by two distinctive features [18]. First, subjects have needs and must carry out activities in order to survive [18]. In the context of the GSD programme these are the clinicians while "carrying out activities" means to interact with objects of the world [19, 20, 23 ]; the young adults with diabetes. An activity is defined as a "[...] "unit of life" of a material subject existing in the objective world." [19]. Activities transform not only objects but also subjects [19].

In other words, an activity is not only influenced by the attributes of the objects but also by the attributes of subjects.

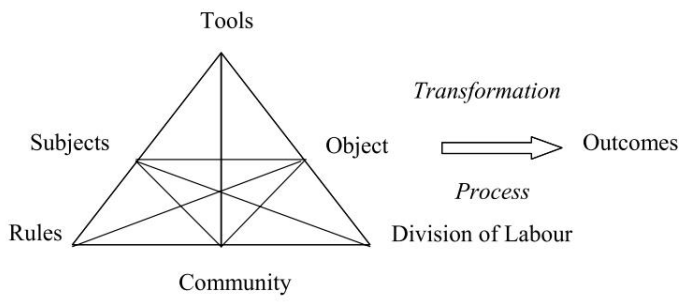

Figure 1: Activity system model of Engeström (adapted from [10])

Building on further literature, Kaptelenin and Nardi summarise five basic principles of activity theory: Object-orientedness, mediation, hierarchical structure of activity, internalisation and externalisation, and development [19].

That human activities are directed towards their objects is stated in the principle of objectorientedness [19]. As stated by Leontiev (1981), an objectless activity is impossible. "Objects motivate and direct activities, around them activities are coordinated, and in them activities are crystallized when the activities are complete." [19].

The principle of mediation states that human activity is mediated by tools. These tools can be external such as a scissor or internal such as concepts or heuristics [22]. As all key distinctive features of humans such as language, society, or culture involve mediation [19], the analysis of tools is necessary to understand human functioning [18].

In summary, the analysis of motivational, goaldirected, and operational aspects of human activity can be analysed based on this model [19]. Given that GSD is focussed on motivating patient with diabetes and providing them with life skills this suggests that Activity theory provides us with a robust and appropriate theoretical lens in which to assess the benefits of the GSD method in the presented pilot study.

\subsection{Contradictions in Activity Theory}

Contradictions in activity systems exist because activities are constantly developing and even mostwell planned actions involve failures, disruptions, and unexpected innovations [19]. By analysing the activity system, the underlying contradictions that lead to these failures, disruptions, or innovations may be recognised [18]. In Activity Theory four types or levels of contradictions can be identified [19].

First-level contradictions deal with inner contradictions of each of the components of an activity system. The components of an activity system are subject, object, community, instruments, rules, and division of labour. For example, a physician chooses a more affordable medication over the best available medication that is more expensive [19].

Second-level contradictions occur between the components of an activity system. For example, if a certain type of medical treatment is unsuitable for certain patients [19].

Third-level contradictions are "potential problems emerging in the relationship between the existing 
forms of an activity system and its potential, more advanced object and outcome" [19].

Fourth-level contradictions occur between different systems of activity that are involved in the production of a joint outcome. For example, a positive effect of surgery can be undermined by an improper follow-up rehabilitation [19].

\section{Research methods}

An exploratory descriptive design undertaken in four stages was used to develop and implement the online GSD program. The stages involved preparing and delivering the GSD program online, and training diabetes educators (DE) in the GSD method and online platform. The setting was Deakin University in Melbourne, Australia.

In Stage 1, a prototype of the GSD program online was developed in collaboration with key diabetes researchers and educators, experienced online educational experts, a web designer and young participants with diabetes. The content and processes of GSD program online were the same as the original written GSD reflection sheets, which are described elsewhere [13]. The GSD program online was accessible using mobile and fixed Apple and Android platforms. The GSD program online sessions (7 in total) were facilitated via Zoom videoconference software (https://zoom.us/,) which is readily and freely accessible. Zoom enabled visual and verbal interactions between participants.

In Stage 2, Zoffmann conducted train-the-trainer workshops in the GSD method. Workshops were held on campus in Melbourne over 2 days in May 2015 for 9 DEs.

In Stage 3, 11 participants and 8 DEs piloted tested the GSD program, which comprised seven conversational sessions over 3 months. Each GSD program online session was facilitated via Zoom between one client and one DE.

Stage 4 involved further training and an evaluation workshop facilitated by Zoffmann at Deakin University in August 2015. Four DEs attended and participants submitted written evaluations. All participants were followed-up individually to clarify and confirm their submitted perspectives about GSD and to gain perspectives of those who did not submit comments. (Dec 2015 - Jan 2016).

\subsection{Participants}

Two participant groups participated: young adults with type 1 diabetes aged 20-39 years and DEs; all of whom provided written informed consent.
Recruitment took place through third party organisations: Australian Diabetes Educator Association (ADEA) and Diabetes Victoria support groups for young people with diabetes. Eleven participants participated. Similarly, DEs based in regional or urban based locations were invited through professional and consumer organisation web sites. Eight DEs consented to participate. Participation involved undertaking two training workshops in the GSD method, conducting the GSD program online (7 sessions) and participating in an evaluation workshop. Thus, 2 DEs conversed with two participants; the remaining 6 DEs each conversed with one client.

\subsection{Data Collection and Analysis}

Following institutional ethics approval (HEAG-H 27_2015) all participants agreed to have all their conversations with the research team digitally recorded for transcription and thematic analysis. Demographic and self-reported skill and experience with online technologies were collected prior to commencement of the GSD program online. At the end of the program the participants, participants and DEs, completed an online anonymous questionnaire about GSD regarding:

1. Their experience using the GSD program (e.g. How did the reflection sheets work for you? What were the benefits? What difficulties, if any, did you experience?).

2. The online delivery of the program, for example: advantages/disadvantages communicating online; cost and time, applicability of the online method to meet their needs.

3. The barriers and facilitators to using communication devices and the GSD program online

Data related to participant experiences of the GSD method and online platform were collected during:

1. fortnightly to monthly meetings between the research team and DEs (during the program of the GSD period)

2. the training workshops for DEs

3. before and during the training and evaluation workshop

4. the final evaluation focus group session Data were audio recorded during these events then transcribed verbatim. Demographic data were subjected to descriptive statistical analysis. Participants' questionnaire responses were thematically analysed using content analysis methods [24].

\section{Results}

Overall the GSD program online was well received by both groups. Findings regarding client perceptions of the program (desire to complete and engage in GSD online) and DE perspectives of 
delivering the GSD program online yielded four main themes. They were: Reflection Created Solutions; Expedient Client Journey; Professional Reward; and GSD on My Terms.

From a utility perspective, participants and DEs provided feedback to improve the technology and web site design.

\subsection{Reflection Created Solutions}

A core element of GSD is deep reflection by the client to identify problems that may be affecting their ability to self-manage their diabetes while negotiating relevant ongoing life events.

Reflections are encouraged and supported by the DE. Although it took time for some participants to formulate the problem, once done, participants led the conversations, which empowered participants and DEs.

DE1 noted: 'Managing diabetes is about more than just the numbers. It's about your personal life more than just the numbers'.

DE3 noted 'It's amazing how you can see some people regularly and never touch on any of these things and then (you discover) that things aren't actually going that well at all and so you can make a plan'

The GSD method guided participants through conversations that facilitated reflection, which benefitted the participants and DEs. The conversations prompted them consider plans for managing diabetes in a more structured way; for example, setting short and long-term goals. The emotional support was important to participants and reflections were enhanced by the flexibility of the program. For example, for participants living in regional areas away from the diabetes education services:

'It (GSD online) helped me to feel like I was receiving emotional support to achieve my goals instead of just focussing on physical aspects of diabetes management. It helped me to consider short and long term goals. I was able to reflect on the bigger picture of my diabetes since diagnosis and to see how well I have managed myself over the last 10 years which I had lost sight of during a recent rough patch. Being able to complete the program in my own home was also extremely convenient for someone who lives in a regional area (Client 01).

\subsection{Expedient Participant Journey}

The GSD program is flexible and timesaving in nature because it can be used 24 hours/7 days a week. Participants often accessed the program outside allocated sessions with their DEs. Most participants worked on reflection sheets in preparation for next session or for their own benefit at times convenient to them. One client was recorded accessing the GSD program reflections sheets more than 40 times outside the scheduled conversations.

Participants indicated the GSD program online saved them time because they could manage competing commitments better, particularly work commitments. Being online meant it reduced the need to take time of work to attend specialist appointments. One client said:

'It takes me 50 minutes each way to get to my usual diabetes educator and I'm a casual worker so I pretty much have to take a whole day off and it's sometimes really hard to get time off work. My diabetes educator is not too bad for time, but quite often when I see specialists I then sit and wait for two and a half hours, and then I'm late for work' (Client 05)

Participants felt the GSD method was focussed and facilitated immediate life changes. Participants reported that practical solutions and outcomes enhanced their sense of control and empowerment. One DE said:

'So working around that problem formulation took a bit of time, and I could see she'd been in [logged in to the online tool] a few times on her own working on it. She made two big decisions, one of them was to go on an insulin pump, which she'd been thinking about for a while, and the other was the decision to move back in with her parents to take some stress away, so that was two practical outcomes' (DE 05)

The flexibility of online GSD meant participants formed a relationships with DEs faster than they would using usual diabetes consultation, which in turn assisted the client's self-management. The DEs were also positive about the flexibility of GSD and the different working relationship enabled by GSD online.

\subsection{Professional Reward}

The DEs indicated that the GSD method encouraged change their approach to a more person- centred plan, which also involved a change in their relationship with their participants. The guided conversations helped the DEs to focus more on the situation from the client's perspective and in turn changed the relationship towards a collaborative approach. 
'It becomes a positive conversation... if you are the person on the other end with diabetes it (must feel like) 'Oh my God, I've got these (blood glucose) readings in front of me.... and you start getting fearful. In this (GSD program), it's more of a help, more positive thinking, and looking at (a collaboration) between the health professional and the person with diabetes, it's a better relationship in this way' (DE 03)

The DEs agreed with the participants' views that the GSD method facilitated conversations that encouraged them to feel more empowered and helped to adopt a more collaborative approach, which helped them to address the participants' issues and find practical strategies to solve the problems.

\subsection{GSD On My Terms}

Participants reported that GSD was particularly attractive to them because it focussed on their needs; GSD was facilitated on their terms. Participants reported they felt 'in control' of the conversations with their DE, and that the conversations were less complicated and more focused. They welcomed the flexibility of GSD and enjoyed using it.

'I have finished all my sessions now, and I really enjoyed them. Each session was relevant for me, and the tool was simple and easy to follow. It didn't try to complicate our discussion, it just made us more focused and gave some direction' (Client 01)

Participants indicated that GSD enabled them to engage differently with DEs. The way GSD program online was implemented shifted the role of 'expert' from the DE to client.

'The way it [GSD] ran was different because usually they [the health professionals] tell you what to do, you have to just tell them about your sugars, how you feel physically and they tell you what to do. 'With my diabetes educator [in the GSD program online] they ask me about my experiences and emotions, and I can suggest things that might work, and find something that actually might work for me instead of something that someone else has come up with' (Client 04)

\subsection{Online Experience and Issues}

Both the participants and DEs reported similar experiences and issues using the technology GSD online. The main issues were system design issues, issues downloading the communication program (Zoom), especially at work, and uncertainty about how to commence using GSD online. Participants were encouraged to contact the web designer/researchers throughout the study whenever required to and to provide feedback either via telephone, emails or during the two workshops.

\subsection{Issues Getting Started}

Initially the participants were given written instructions about to how to access the online conference software (Zoom). The web designer was online to support the participants and DEs and talk them through the access process. One participant highlighted some difficulties with the way the written instructions were presented.

'I didn't see that I had to set up Zoom and so we set up the meeting with my diabetes educator and my boyfriend had gone to basketball and I had no one to ask. 'I didn't know how to upload Zoom. If it had been in the first five steps instead of rather than at the bottom of the email maybe I would have been ready to go when I started the first session' (Client 01)

It became apparent that a face-to-face session to explain how to use the technology was warranted to enable a smooth set-up process for participants and DEs to avoid wasting time and causing frustration, which could have jeopardised the study. Participants who attended on-site-sessions did not experience access problems.

\subsection{Design Issues}

The initial learning curve was very steep for both groups. Adherence to the paper based GSD system was necessary to keep the GSD training consistent. The online GSD application therefore only included limited "web style" finesses that the young participants are used to; hence application alterations were required in the early stages of implementation. Alterations were expected in a pilot study, but were nevertheless challenging at times for participants.

More on-going issues related to design issues such as a session not saving, meant delays in progression of the program, and frustration when sessions had to be repeated.

I had trouble editing, I could not edit the timeline, I wanted to add something in, but it wiped my information and I had to enter it again, that was a bit tedious' (Client 01)

At times participants were able to solve issues themselves, which was regarded as a positive experience. 
'I'll just see (if I can set up Zoom) and I did it and it was really easy, so that was satisfying' (Client 01)

'...the big handprint was covering the login page, could not see past it (on the iPhone)... but when turned the phone on landscape, it was ok.' (Client 02)

Researchers responded very quickly to client feedback. The team recorded short videos that recapped the purpose of each conversation to guide participants smoother through the program. Participants and DEs were able to watch a 2 minute video prior to commencing, which they regarded favourably because it reduced concerns about remembering the content of sessions accurately.

\subsection{Saving Time and Free of Cost}

A significant benefit of the GSD program online was that it was free to participants and saved time for participants by reaching solutions to their identified issues rapidly. It was surprising how effective the conversations were due to the reflective preparations undertaken by the participants and DEs.

\section{'It was a little bit daunting with the sessions, but it turns out that none of them went for an hour' (Client 01) \\ 'I was thinking if we want to make it workable we are better to use a free systems if we can, because that's a barrier for a lot of people' (DE 02)}

Participants demonstrated resilience when the technology didn't work optimally. One outcome of the design issue was the willingness to share technology solutions among participants.

\section{Discussion and Conclusions}

Our findings showed that the GSD program is transferrable to an online platform and was readily accepted by both DEs and participants in an online form. Indeed the findings show GSD online was preferable to traditional face-to-face consultations for young adults with T1DM, especially if they had little access to health services due to geographic distances or lost motivation.

The online version of GSD improved participants' diabetes self-management, which is likely to improve outcomes. Client confidence was enhanced through timely and meaningful decisions agreed upon with DE at each GSD session. GSD in this context when framed in the perspective of activity theory is then an extern mediation of the human activities around diabetes self-management, and helps people to self-manage better by making them more empowered. Our results indicated that participants and DEs regarded the GSD program online as a tool to empower participants to make their decisions and solve problems on their own terms according to individual priorities.

Furthermore, we note that even though this was a small pilot study to establish proof of concept, we identified directional data that serves to show the possibility of responses from one participant becoming generalizable to other similarly situated participants and believe that this might be a further benefit of the system in assisting participants with diabetes or other chronic conditions. In addition this would provide DEs with learning for managing their participant cohort groups; i.e. single loop , double loop and even triple loop learning might be supporter. Clearly, we need to test for this in our future studies which is part of our next steps.

Decision making and problem solving are powerful self-management strategies and health professionals and participants are repeatedly involved in these activities in all clinical settings. From an activity theory perspective, activities typically include decision making and problem solving and the resolution of problems is always focused on the goal [19]; in this case, better selfmanagement which in turn leads to tighter glucose control. Solving. Zoffmann and Kirkevold [13, 25] argued that using GSD is beneficial for patients and health professionals because GSD is grounded in theory and evidence based. Thus, GSD is tailored to achieving change and targets unproductive behaviour patterns observed in other studies that focus on decision making and problem solving strategies between patients and professionals [2527]. Our study demonstrated that the online version of GSD effectively developed confidence in participants and DEs to work in partnership to share decision making and problem-solving; hence, GSD empowered both parties.

Most participants accessed GSD online multiple times between scheduled appointments and both groups left information online anytime they desired. This practice could be considered a 'brain dump' and was regarded as being very important to the reflection and decision making processes. The online GSD program captured client information that may have otherwise not been shared with DEs and could be used to inform management strategies. Recording reflections online was very useful because rereading the reflection sheets multiple times helped participants and DEs see patterns of behaviours, recurring issues or difficulties in lifestyles. It also became evident that the patterns of behaviours and life style issues were similar across all participants and also transferable to other chronic conditions Health professionals need detailed knowledge of the barriers and 
enablers of self-management to empower participants. Health professionals also need to understand their own roles in these barriers/enablers, find ways to overcome them, and acknowledge when common goals were achieved $[25,27]$.

We involved participants, young people with diabetes, from the commencement of the study because involving end-users in the design is crucial. Client documentation on the sites provided insight that helped the DEs and researchers understand the client needs, which, according to Kayser, Kushniruk, Osborne, Norgaard, and Turner [28] is critical to develop a usable and effective online product and system. In the health care context, Kayser et al. provided a framework comprising user dimensions and a task dimension. These dimensions emphasise the need for endusers/participants to have good knowledge about their own health, and the ability to engage with technology that it is beneficial, secure and controllable. End-users/participants need access to technologies that work and suit their needs. All these factors need to be considered when developing electronic programs.

The time saving nature of the online GSD method was important to participants. Results suggest participants and DEs were prepared for the conversations and moved forward and backward between documents together during conversation sessions.

Completing the reflection sheets kept participants on track and facilitated their conversations with the $\mathrm{DE}$ about changes they were making. This was a similar finding in other studies of a face-to-face GSD with paper-based reflection sheets [2, 27] and aligned with the purpose of GSD method; that is, to discover and express personal difficulties and priorities related to the chronic conditions enable people to discover their potential for change [29]. In our study, online conversations generally were shorter, more focussed and at times more frequent, compared to face-to-face GSD using paper-based reflection sheets. This suggests that an online GSD version will further the translation of changes into practice. In terms of Activity theory, the new technology represents the tool which is introduced with the aim to assist subjects and objects interact and conduct all activities better in order to attain the goal; ie a successful outcome which is in this case tight blood sugar and better self-management.

An important finding of our study was how essential training DEs was to enable them to use the GSD method effectively. The DEs who had 2 participants found the second client's program was easier to conduct. Technology issues distracted some from the main purpose of the conversations at times. From an Activity theory perspective, this is a typical third level contradiction given that the technology tool is introduced with the aim to enable better alignment and support to attain the goal/outcome [19]; however the reactions to the new technology or enhanced object and outcomes over the existing activities had a different effect. One DE found it nerve-wracking to use new technology at the same as a using a new therapeutic method. It is therefore of outmost importance to ensure health professionals receive thorough training in GSD program methods as well as computer skills. Other researchers who addressed the model of building capabilities using technologies also indicated that it is integral for users to believe the technology is beneficial, engaging and can be controlled [28].

Translating the GSD program to an online platform clearly helped participants improve their capacity to self-monitor their health and deliver access to a highly responsive health care system.

\subsection{Conclusions}

Transferability of the GSD method to an online platform provided demotivated and geographically isolated participants with a novel, inexpensive and readily accessible therapeutic intervention to improve their diabetes self-management. The GSD program online improved participants' diabetes self-management and communication between DEs and participants. Further development and inclusion of new technologies is required for the GSD platform to fully realise its capacity to engage participants to improve diabetes self-management and health outcomes. As with the face-to-face GSD method, health professionals using the GSD program online require extensive education and training in GSD methods to optimise their capacity to use the program to competently to help participants realise their diabetes self-management potential.

From the perspective of Activity theory we can see that the GSD method enables via the tool (the online system) subjects (clinicians) and objects (patients) to not just interact better but through these interactions or conversations develop a better understanding of critical contextual aspects that impact the realisation of the goal; namely sustained and continuous tighter management of blood glucose levels. Specifically, the tool assists the activities between the subject and object to interact at a better level so that the outcomes and goals are more readily achieved. We note however that third level contradictions were observed. The identification of such contradictions enables them to be addressed expeditiously so that the new technology (tool) can be further modified to ensure that its merits are not diminished by the 
development of such contradictions. In the GSD context, this is addressed by focussing on enhancing the training elements of the health professionals.

In the literature, it is recognised that Activity theory can be a complex framework for analysing and designing purposes. Even though it is characterised as an analytical framework, it is recognised that it does not offer ready-made techniques and procedures for research. It is considered as an evolving framework that only contains general guidelines and therefore must be further developed [30]. However, Activity theory is regarded as a powerful lens for studying complex social systems. This statement can be underpinned by the fact that Activity theory is focusing on the relationships that exist in an activity system. In addition, the historical development of the activity as well as the use of tools is analysed. Different user perspectives of the subjects are considered, too $[30,31]$. This has been our experience with using Activity theory and we call for more incorporation of this theory when examining technology enabled and supported healthcare scenarios.

\section{Conflict of interest}

The researchers have no conflict of interest to declare.

\section{Acknowledgements}

We sincerely thank all participating young adults and diabetes educators for their time and for sharing their experiences, Professor Lars Kayser, University of Copenhagen and Professor Vibeke Zoffmann, Rigshospitalet and University of Copenhagen for their expert inputs, Bill Haig, Monash University for designing the web site platform Diabetes Victoria staff, Caroline Hines and Karen Crawford for supporting and promoting the project, Educational Developer Dr Ian Story, Faculty of Health, Deakin University for constructive consultations, Research Fellow Elizabeth Stewart for project management, Australian Diabetes Educator Association Victoria for support of advertising the project. We thank and acknowledge funding from the Centre for Quality and Patient Safety Research (QPS), Deakin University that made it all possible.

\section{References}

[1] Peyrot, M., et al., Diabetes Attitudes Wishes and Needs 2 (DAWN2): a multinational, multistakeholder study of psychosocial issues in diabetes and person-centred diabetes care.
Diabetes Res Clin Pract, 2013. 99(2): p. 174-84.

[2] Zoffmann, V., D. Vistisen, and M. Due Christensen, A cross-sectional study of glycaemic control, complications and psychosocial functioning among 18- to 35year-old adults with Type 1 diabetes. Diabetic Medicine, 2014. 31(4): p. 493499.

[3] Polonsky, W.H., et al., Assessing psychosocial distress in diabetes: development of the diabetes distress scale. Diabetes Care, 2005. 28(3): p. 626-31.

[4] International Diabetes Federation, IDF Diabetes Atlas. 2014, Brussels: IDF.

[5] Zoffmann, V., Guided Self-Determination: A Life Skills Approach Developed In Difficult Type 1 Diabetes in Department Of Nursing Science. 2004, University of Aarhus: Denmark.

[6] Zoffmann, V. and T. Lauritzen, Guided selfdetermination improves life skills with type 1 diabetes and AlC in randomized controlled trial. Patient Educ Couns, 2006. 64(1-3): p. 78-86.

[7] NDSS. National Diabetes Services Scheme (NDSS) Statistical Snapshot. 201621 February 2017]; Available from: http://www.ndss.com.au/en/Research/Data -Snapshots

[8] Lauritzen, T. and V. Zoffmann, Understanding the psychological barriers to effective diabetes therapy. Diabetes Voice 2004. 49: p. 16-18.

[9] Kadirvelu, A., S. Sadasivan, and S.H. Ng, Social support in type II diabetes care: a case of too little, too late. Diabetes Metab Syndr Obes, 2012. 5: p. 407-17.

[10] Mamykina, L., et al. MAHI: Investigation of Social Scaffolding for Reflective Thinking in Diabetes Management. 2008. Florence.

[11] Rasmussen, B., et al., Young Women With Type 1 Diabetes' Management of Turning Points and Transitions. Qualitative Health Research, 2007. 17(3): p. 300-310.

[12] Rasmussen, B., et al., Young adults' management of Type 1 diabetes during life transitions. J Clin Nurs, 2011. 20(13-14): p. 1981-92.

[13] Zoffmann, V. and M. Kirkevold, Relationships and their potential for change developed in difficult type 1 diabetes. Qualitative Health Research, 2007. 17(5): p. 625-638.

[14] Dunning, T. and S. Salvage, Information needs of young adults with type 2 diabetes: $a$ literature review. Australia Diabetes Educator Magazine 2013. 16(1): p. 19-25.

[15] Rasmussen, B., P. Dunning, and B. O'Connell, Young women with diabetes: using Internet communication to create stability 
during life transitions. Journal of Clinical Nursing, 2007. 16(3a): p. 17-24.

[16] Sensis and Australian Interactive Media Industry Association. Yellow ${ }^{\mathrm{TM}}$ social media report: What Australian people and businesses are doing with social media. 2012; Available from: about.sensis.com.au/IgnitionSuite/uploads/ docs/FinalYellow_SocialMediaReport_dig ital_screen.pdf.

[17] Uden, L. and N. Willis. Designing User Interfaces using Activity Theory. in 34th Hawaii International Conference on System Sciences. 2011. Hawaii.

[18] Engeström, Y., R. Miettinen, and R.L. Punamäki, Perspectives on Activity Theory. 1999, Cambridge Cambridge University Press.

[19] Kaptelinin, V. and B. Nardi, Synthesis Lectures on Human-Centered Informatics: Activity Theory in HCI: Fundamentals and Reflections. 2012, Morgan \& Claypool Publishers: San Rafael, CA, USA.

[20] Mwanza, D. and Y. Engeström, Pedagogical Adeptness in the Design of E-learning Environments: Experiences from the Lab@Future Project. 2003, Center for Activity Theory \& Developmental Work Research, University of Helsinki: Finland.

[21] Bedny, G.Z., Application of SystemicStructural Activity Theory to Design and Training. 2014: CRC Press.

[22] Nardi, B.A., Context and consciousness: activity theory and human-computer interaction. 1996: MIT Press.

[23] Kaptelinin, V., Activity Theory, in The Encyclopedia of Human-Computer Interaction, M.a.D. Soegaard, Rikke Friis, Editor. 2014, The Interaction Design Foundation: Aarhus, Denmark.

[24] Elo, S. and H. Kyngas, The qualitative content analysis process. J Adv Nurs, 2008. 62(1): p. 107-15.

[25] Zoffmann, V. and M. Kirkevold, Life versus disease in difficult diabetes care: conflicting perspectives disempower patients and professionals in problem solving. Qual Health Res, 2005. 15(6): p. 750-65.

[26] Zoffmann, V., I. Harder, and M. Kirkevold, A person-centered communication and reflection model: sharing decision-making in chronic care. Qual Health Res, 2008. 18(5): p. 670-85.

[27] Zoffmann, V. and M. Kirkevold, Realizing empowerment in difficult diabetes care: a guided self-determination intervention. Qual Health Res, 2012. 22(1): p. 103-18.

[28] Kayser, L., et al., Enhancing the Effectiveness of consumer-focused health information technology systems through ehealth literacy: a framework for understanding users' needs. JMIR Human Factors, 2015. 2(1): p. e9.

[29] Zoffmann, V., Å. Hörnsten, and S. Storbækken, Translating person-centred care into practice: a comparative analysis of motivational interviewing, illnessintegration support, and guided selfdetermination. Patient Educ Couns 2016. 99(3): p. 400-7.

[30] Greenhow, C. and B. Belbas, Using activityoriented design methods to study collaborative knowledge-building in elearning courses within higher education. International Journal of ComputerSupported Collaborative Learning, 2007. 2(4): p. 363-391.

[31] Sadeghi, P., P. Andreev, and M. Benyoucef. Activity Theory Driven System Analysis Of Complex Healthcare Processes. in European Conference on Information Systems. 2014. Tel Aviv, Israel. 